PROCEEDINGS OF THE

AMERICAN MATHEMATICAL SOCIETY

Volume 138, Number 8, August 2010, Pages 2979-2990

S 0002-9939(10)10302-5

Article electronically published on April 1, 2010

\title{
HEWITT-MARCZEWSKI-PONDICZERY TYPE THEOREM FOR ABELIAN GROUPS AND MARKOV'S POTENTIAL DENSITY
}

\author{
DIKRAN DIKRANJAN AND DMITRI SHAKHMATOV
}

(Communicated by Alexander N. Dranishnikov)

\begin{abstract}
For an uncountable cardinal $\tau$ and a subset $S$ of an abelian group $G$, the following conditions are equivalent:

(i) $|\{n s: s \in S\}| \geq \tau$ for all integers $n \geq 1$;

(ii) there exists a group homomorphism $\pi: G \rightarrow \mathbb{T}^{2^{\tau}}$ such that $\pi(S)$ is dense in $\mathbb{T}^{2^{\tau}}$.

Moreover, if $|G| \leq 2^{2^{\tau}}$, then the following item can be added to this list:

(iii) there exists an isomorphism $\pi: G \rightarrow G^{\prime}$ between $G$ and a subgroup $G^{\prime}$ of $\mathbb{T}^{2^{\tau}}$ such that $\pi(S)$ is dense in $\mathbb{T}^{2^{\tau}}$.

We prove that the following conditions are equivalent for an uncountable subset $S$ of an abelian group $G$ that is either (almost) torsion-free or divisible:

(a) $S$ is $\mathscr{T}$-dense in $G$ for some Hausdorff group topology $\mathscr{T}$ on $G$;

(b) $S$ is $\mathscr{T}$-dense in some precompact Hausdorff group topology $\mathscr{T}$ on $G$;

(c) $|\{n s: s \in S\}| \geq \min \left\{\tau:|G| \leq 2^{2^{\tau}}\right\}$ for every integer $n \geq 1$.

This partially resolves a question of Markov going back to 1946 .
\end{abstract}

We use $\mathbb{N}$ and $\mathbb{P}$ to denote the set of positive natural numbers and prime numbers, respectively. The symbol $\mathfrak{c}$ denotes the cardinality of the continuum, and $\omega$ denotes the first infinite cardinal. For an infinite cardinal $\kappa$, let

$$
\log \kappa=\min \left\{\tau \geq \omega: \kappa \leq 2^{\tau}\right\} .
$$

The group of integer numbers is denoted by $\mathbb{Z}$.

For a subset $X$ of an abelian group $G$, we use $\langle X\rangle$ to denote the subgroup of $G$ generated by $X$, and we let $n X=\{n x: x \in X\}$ for every $n \in \mathbb{Z}$. As usual,

$$
t(G)=\{g \in G: n g=0 \text { for some } n \in \mathbb{N}\}
$$

denotes the torsion part of $G$. An abelian group $G$ is said to be:

(i) torsion-free if $t(G)=\{0\}$;

(ii) torsion if $t(G)=G$;

(iii) bounded if $n G=\{0\}$ for some $n \in \mathbb{N}$;

(iv) divisible if $n G=G$ for every $n \in \mathbb{N}$.

Received by the editors November 10, 2008, and, in revised form, November 24, 2009.

2010 Mathematics Subject Classification. Primary 22A05; Secondary 20K99, 22C05, 54A25, 54B10, 54D65.

Key words and phrases. Abelian group, monomorphism, homomorphism, potentially dense set, dense subset, precompact group.

The first author was partially supported by SRA, grants P1-0292-0101 and J1-9643-0101 and by grant MTM2009-14409-C02-01.

The second author was partially supported by Grant-in-Aid for Scientific Research (C) No. 19540092 of the Japan Society for the Promotion of Science (JSPS).

(C)2010 American Mathematical Society 2979

Reverts to public domain 28 years from publication 
Recall that a group homomorphism $\varphi: G \rightarrow H$ from an abelian group $G$ to an abelian group $H$ is a monomorphism if $\operatorname{ker} \varphi=\{0\}$.

A topological group is precompact if it is isomorphic to a subgroup of some compact Hausdorff group. As usual, $w(X)$ denotes the weight of a topological space $X$.

\section{INTRODUCTION}

The classical result of Hewitt-Marczewski-Pondiczery states: If $\tau$ is an infinite cardinal, $I$ is a set such that $|I| \leq 2^{\tau}$, and for every $i \in I$ a space $X_{i}$ has a dense subset of size $\leq \tau$, then the product $X=\prod\left\{X_{i}: i \in I\right\}$ also has a dense subset of size $\leq \tau$ (see, for example, [8, Theorem 2.3.15]). In this paper we investigate the following "algebraic version" of this theorem. Let $\kappa$ be an infinite cardinal and $\mathbb{T}=\mathbb{R} / \mathbb{Z}$ be the circle group. Given a fixed subset $S$ of an abelian group $G$, we attempt to find a group homomorphism $\pi: G \rightarrow \mathbb{T}^{\kappa}$ such that $\pi(S)$ becomes dense in $\mathbb{T}^{\kappa}$. Of particular interest is the special case when $\pi$ can be chosen to be a monomorphism, that is, when the group $G$ and the subgroup $\pi(G)$ of $\mathbb{T}^{\kappa}$ become isomorphic. Our choice of the target group is justified by the fact that every abelian group $G$ is isomorphic to a subgroup of $\mathbb{T}^{\kappa}$ for a suitable cardinal $\kappa$. To ensure a closer resemblance to the Hewitt-Marczewski-Pondiczery theorem, we pay special attention to the case $\kappa=2^{\tau}$ for some infinite cardinal $\tau$ by addressing the following question: Given a subset $S$ of an abelian group $G$ such that $|S| \geq \tau$ and $|G| \leq 2^{2^{\tau}}$, does there exist a monomorphism $\pi: G \rightarrow \mathbb{T}^{2^{\tau}}$ such that $\pi(S)$ becomes dense in $\mathbb{T}^{2^{\tau}}$ ? We completely resolve this problem in the case when $S$ is uncountable. Moreover, we provide a complete answer to this problem even for a countable set $S$ such that no multiple $n S$ of $S$ (for $n \in \mathbb{N}$ ) is contained in a finitely generated subgroup of $G$. The remaining case is being resolved by the authors in [6].

The origin of this setting can be traced back to the 1916 paper of Weyl [11. We recall the classical Weyl's uniform distribution theorem: Given a faithfully indexed set $S=\left\{a_{n}: n \in \mathbb{N}\right\} \subseteq \mathbb{Z}$, the set of all $\alpha \in \mathbb{T}$ such that the set $S \alpha=\left\{a_{n} \alpha: n \in \mathbb{N}\right\} \subseteq \mathbb{T}$ is uniformly distributed has full measure 1 . Since uniform distribution implies density in $\mathbb{T}$, it follows that $S \alpha$ is dense in $\mathbb{T}$ for almost all $\alpha \in \mathbb{T}$. Every $\alpha \in \mathbb{T}$ uniquely determines a homomorphism $h_{\alpha}: \mathbb{Z} \rightarrow \mathbb{T}$ such that $h_{\alpha}(1)=\alpha$. Furthermore, $\alpha \in \mathbb{T}$ generates a dense subgroup $\langle\alpha\rangle$ of $\mathbb{T}$ iff $\alpha$ is non-torsion iff the homomorphism $h_{\alpha}$ is a monomorphism. Hence, one can state (a consequence of) Weyl's theorem by simply saying that for every infinite subset $S$ of $\mathbb{Z}$, there exists a monomorphism $\pi: \mathbb{Z} \rightarrow \mathbb{T}$ such that $\pi(S)$ is dense in $\mathbb{T}$. Tkachenko and Yaschenko 10 consider homomorphisms $\pi: G \rightarrow \mathbb{T}^{\omega}$ such that $\pi(S)$ is dense in $\mathbb{T}^{\omega}$, for a certain class of groups $G$. They use such homomorphisms as a technical tool in addressing the problem suggested first in 1946 by Markov [9].

According to Markov [9], a subset $S$ of a group $G$ is called potentially dense (in $G$ ) provided that $G$ admits some Hausdorff group topology $\mathscr{T}$ such that $S$ is dense in $(G, \mathscr{T})$. The last section of $[9]$ is exclusively dedicated to the following problem: Which subsets of a group $G$ are potentially dense in $G$ ? Markov [9] proved that every infinite subset of $\mathbb{Z}$ is potentially dense in $\mathbb{Z}$. This was strengthened in [7, Lemma 5.2] by showing that every infinite subset of $\mathbb{Z}$ is dense in some precompact metric group topology on $\mathbb{Z}$. (Apparently, the authors of [9] and [7] were unaware that both these results easily follow from Weyl's uniform distribution theorem.) Further progress was obtained by Tkachenko and Yaschenko [10, who proved the 
following theorem: If an abelian group $G$ of size at most $\mathfrak{c}$ is either almost torsionfree or has exponent $p$ for some prime $p$, then every infinite subset of $G$ is potentially dense in $G$. (According to [10], an abelian group $G$ is almost torsion-free if $r_{p}(G)$ is finite for every prime $p$.)

In [6], the authors resolved Markov's problem for countable sets: A countable subset $S$ of an abelian group $G$ is potentially dense in $G$ if and only if $|G| \leq 2^{\text {c }}$ and $S$ is Zariski dense in $G$. Recall that a subset $S$ of an abelian group $G$ is said to be Zariski dense in $G$ provided that if $k \in \mathbb{N}, g_{1}, g_{2}, \ldots, g_{k} \in G, n_{1}, n_{2}, \ldots, n_{k} \in \mathbb{N}$ and each $s \in S$ satisfies the equation $n_{i} s=g_{i}$ for some $i=1,2, \ldots, k$ (depending on $s$ ), then every $g \in G$ also satisfies some equation $n_{j} g=g_{j}$, for a suitable $j=1,2, \ldots, k$ ([5]; see also [4, Section 5]).

In this paper we investigate the remaining case of uncountable sets. In particular, we obtain a new sufficient condition that guarantees that a subset $S$ of an abelian group $G$ is potentially dense in $G$. Moreover, when this condition is satisfied, we prove that the topology $\mathscr{T}$ on $G$ such that $S$ is $\mathscr{T}$-dense in $G$ can be chosen to be precompact. When $S$ is uncountable and $G$ belongs to a wide class of abelian groups (for example, almost torsion-free or divisible groups), our sufficient condition turns out to be also necessary for the potential density of $S$ in $G$. And last but not least, our sufficient condition is rather powerful in the countable case as well, because the only case that is not covered by it is when $n S$, for a suitable $n \in \mathbb{N}$, is contained in a finitely generated subgroup of $G$. Therefore, it is only this special case that still requires the substantially more sophisticated techniques from [6] to prove potential density (in some precompact group topology).

\section{Sending a given subset of an Abelian group Densely in $\mathbb{T}^{\kappa}$}

Definition 2.1. Let $\tau$ be an infinite cardinal. We say that a subset $S$ of an abelian group $G$ is $\tau$-wide if $n S \backslash\left\langle S^{\prime}\right\rangle \neq \emptyset$ for every $n \in \mathbb{N}$ and each $S^{\prime} \subseteq S$ with $\left|S^{\prime}\right|<\tau$.

Our next proposition collects four simple facts that clarify the above definition and facilitate future references.

Proposition 2.2. Let $S$ be a subset of an abelian group $G$.

(i) If $S$ is uncountable, then $S$ is $\tau$-wide if and only if $\min \{|n S|: n \in \mathbb{N}\} \geq \tau$.

(ii) If $S$ contains an infinite independent subset $S^{\prime}$, then $S$ is $\left|S^{\prime}\right|$-wide.

(iii) If $G$ is torsion, then $S$ is $\omega$-wide if and only if $n S$ is infinite for every $n \in \mathbb{N}$ (that is, $S$ is almost 0-torsion in the sense of [3]). Therefore, $G$ contains an $\omega$-wide set $S$ if and only if $G$ is unbounded (and in such a case the $\omega$-wide sets coincide with the Zariski dense sets).

(iv) If $S$ is not $\omega$-wide, then $n S$ is contained in a finitely generated subgroup of $G$, for a suitable $n \in \mathbb{N}$.

Theorem 2.3. Let $\tau$ be an infinite cardinal and $S$ be a $\tau$-wide subset of an abelian group $G$. Then there exist a subgroup $H$ of $G$ and a monomorphism $\varphi: H \rightarrow \mathbb{T}^{2^{\tau}}$ such that $|H|=\tau$ and $\varphi(H \cap S)$ is dense in $\mathbb{T}^{2^{\tau}}$.

The proof of this theorem is postponed until Section 5 .

Corollary 2.4. Let $\tau$ be an infinite cardinal and $S$ be a $\tau$-wide subset of an abelian group $G$. Then:

(i) there exists a group homomorphism $\pi: G \rightarrow \mathbb{T}^{2^{\tau}}$ such that $\pi(S)$ is dense in $\mathbb{T}^{2^{\tau}}$; 
(ii) if one additionally assumes that $|G| \leq 2^{2^{\tau}}$, then $\pi$ from the item (i) can be chosen to be a monomorphism.

Proof. Let $H$ and $\varphi$ be as in the conclusion of Theorem 2.3. The proof now branches into two cases, depending on which item of our corollary holds.

(i) Since $\mathbb{T}^{2^{\tau}}$ is a divisible group, there exists a group homomorphism $\pi: G \rightarrow$ $\mathbb{T}^{2^{\tau}}$ extending $\varphi$.

(ii) Since $|H|=\tau<2^{2^{\tau}}$ and $r_{p}(G) \leq|G| \leq 2^{2^{\tau}}=\left|\mathbb{T}^{2^{\tau}}\right|=r_{p}\left(\mathbb{T}^{2^{\tau}}\right)$ for every $p \in \mathbb{P} \cup\{0\}$, we can extend $\varphi$ to a monomorphism $\pi: G \rightarrow \mathbb{T}^{2^{\tau}}$; see [3, Lemma 3.17].

Returning back to the common part of the proof, note that $\varphi(H \cap S) \subseteq \pi(S)$ and $\varphi(H \cap S)$ is dense in $\mathbb{T}^{2^{\tau}}$, so $\pi(S)$ must be dense in $\mathbb{T}^{2^{\tau}}$ as well.

Theorem 2.5. Let $\kappa$ be a cardinal such that $\kappa>\mathfrak{c}$, and let $\tau=\log \kappa$. For a subset $S$ of an abelian group $G$, the following conditions are equivalent:

(i) there exists a group homomorphism $\varpi: G \rightarrow \mathbb{T}^{\kappa}$ such that $\varpi(S)$ is dense in $\mathbb{T}^{\kappa}$;

(ii) $\tau \leq \min \{|n S|: n \in \mathbb{N}\}$;

(iii) $S$ is $\tau$-wide.

Proof. (i) $\rightarrow$ (ii) Let $n \in \mathbb{N}$. Since $\varpi(S)$ is dense in $\mathbb{T}^{\kappa}, w\left(n \mathbb{T}^{\kappa}\right) \leq 2^{|n S|}$ by Lemma 3.1(ii) below. Since $\mathbb{T}^{\kappa}$ is divisible, $n \mathbb{T}^{\kappa}=\mathbb{T}^{\kappa}$, and so $\kappa=w\left(\mathbb{T}^{\kappa}\right) \leq 2^{|n S|}$, which yields $\tau=\log \kappa \leq|n S|$. This proves (ii).

(ii) $\rightarrow$ (iii) Since $\kappa>\mathfrak{c}, \tau$ is uncountable. Applying (ii) with $n=1$, we conclude that $S$ is uncountable. Then $S$ is $\tau$-wide by (ii) and Proposition 2.2(i).

(iii) $\rightarrow$ (i) Let $\pi$ be as in the conclusion of Corollary 2.4(i). From $\tau=\log \kappa$ it follows that $\kappa \leq 2^{\tau}$. Let $\psi: \mathbb{T}^{2^{\tau}} \rightarrow \mathbb{T}^{\kappa}$ be the projection on the first $\kappa$ coordinates. Since $\psi$ is a homomorphism, so is $\varpi=\psi \circ \pi: G \rightarrow \mathbb{T}^{\kappa}$. Since $\psi$ is continuous and $\pi(S)$ is dense in $\mathbb{T}^{2^{\tau}}$, the set $\psi(\pi(S))=\varpi(S)$ is dense in $\psi\left(\mathbb{T}^{2^{\tau}}\right)=\mathbb{T}^{\kappa}$.

Theorem 2.6. Let $\kappa$ be a cardinal such that $\kappa>\mathfrak{c}$, and let $\tau=\log \kappa$. For a subset $S$ of an abelian group $G$, the following conditions are equivalent:

(i) there exists a monomorphism $\varpi: G \rightarrow \mathbb{T}^{\kappa}$ such that $\varpi(S)$ is dense in $\mathbb{T}^{\kappa}$;

(ii) $|G| \leq 2^{\kappa}$ and $\tau \leq \min \{|n S|: n \in \mathbb{N}\}$;

(iii) $|G| \leq 2^{\kappa}$ and $S$ is $\tau$-wide.

Proof. (i) $\rightarrow$ (ii) Clearly, $|G|=|\varpi(G)| \leq\left|\mathbb{T}^{\kappa}\right|=2^{\kappa}$. The other inequality in item (ii) follows from the implication (i) $\rightarrow$ (ii) of Theorem 2.5 .

(ii) $\rightarrow$ (iii) follows from the implication (ii) $\rightarrow$ (iii) of Theorem 2.5 .

(iii) $\rightarrow$ (i) Let $H$ and $\varphi$ be as in the conclusion of Theorem 2.3. From $\tau=\log \kappa$ it follows that $\tau \leq \kappa \leq 2^{\tau}$.

For every $h \in H \backslash\{0\}$, there exists $\xi_{h} \in 2^{\tau}$ such that $\varphi(h)\left(\xi_{h}\right) \neq 0$. Define $\Xi=\left\{\xi_{h} \in 2^{\tau}: h \in H \backslash\{0\}\right\} \subseteq 2^{\tau}$. Let $\mu: \mathbb{T}^{2^{\tau}} \rightarrow \mathbb{T}^{\Xi}$ be the projection. Then $\mu \uparrow_{\varphi(H)}: \varphi(H) \rightarrow \mathbb{T}^{\Xi}$ is a monomorphism. Since $|\Xi| \leq|H \backslash\{0\}| \leq|H|=\tau \leq \kappa$, it follows that there exists a continuous surjective homomorphism $\psi: \mathbb{T}^{2^{\tau}} \rightarrow \mathbb{T}^{\kappa}$ such that $\chi=\psi \circ \varphi: H \rightarrow \mathbb{T}^{\kappa}$ is a monomorphism. Since $\psi$ is continuous and $\varphi(H \cap S)$ is dense in $\mathbb{T}^{2^{\tau}}$, the set $\psi(\varphi(H \cap S))=\chi(H \cap S)$ is dense in $\psi\left(\mathbb{T}^{2^{\tau}}\right)=\mathbb{T}^{\kappa}$.

Since $|H|=\tau \leq \kappa<2^{\kappa}$ and $r_{p}(G) \leq|G| \leq 2^{\kappa}=\left|\mathbb{T}^{\kappa}\right|=r_{p}\left(\mathbb{T}^{\kappa}\right)$ for every $p \in \mathbb{P} \cup\{0\}$, we can extend $\chi$ to a monomorphism $\varpi: G \rightarrow \mathbb{T}^{\kappa}$; see [3. Lemma 3.17]. Since $\chi(H \cap S) \subseteq \varpi(S)$ and $\chi(H \cap S)$ is dense in $\mathbb{T}^{\kappa}$, the set $\varpi(S)$ must be dense in $\mathbb{T}^{\kappa}$ as well. 
The counterpart of Theorems 2.5 and 2.6 for $\kappa \leq \mathfrak{c}$ is proved in [6].

\section{MARKOV'S POTENTIAL DENSITY}

We start this section with a simple necessary condition for potential density.

Lemma 3.1. Let $S$ be a dense subset of a Hausdorff group $G$. Then:

(i) for every $n \in \mathbb{N}$, the set $n S$ is dense in the subgroup $n G$ of $G$;

(ii) $w(n G) \leq 2^{|n S|}$ and $|n G| \leq 2^{2^{|n S|}}$ for each $n \in \mathbb{N}$.

Proof. (i) The map $g \mapsto n g$ that sends $G$ to the subgroup $n G$ of $G$ is continuous. Since $S$ is dense in $n G, n S$ must be dense in $n G$.

(ii) Let $n \in \mathbb{N}$. From (i) we conclude that $n S$ is a dense subset of the Tychonoff space $n G$. Therefore, $w(n G) \leq 2^{|n S|}$ by [8, Theorem 1.5.7] and $|n G| \leq 2^{2^{|n S|}}$ by $[8$, Theorem 1.5.3].

Corollary 3.2. If $S$ is a potentially dense subset of a group $G$, then

$$
\log \log |n G| \leq|n S| \text { for all } n \in \mathbb{N} \text {. }
$$

Our next theorem provides a general sufficient condition for potential density that also allows it to be realized by some precompact group topology.

Theorem 3.3. Let $\tau$ be an infinite cardinal and $S$ be a $\tau$-wide subset of an abelian group $G$ such that $|G| \leq 2^{2^{\tau}}$. Then there exists a precompact Hausdorff group topology $\mathscr{T}$ on $G$ such that $S$ is dense in $(G, \mathscr{T})$.

Proof. Let $\pi: G \rightarrow \mathbb{T}^{2^{\tau}}$ be a monomorphism from Corollary 2.4(ii), and let $\mathscr{T}^{\prime}$ be the topology $\pi(G)$ inherits from $\mathbb{T}^{2^{\tau}}$. Then $\left(\pi(G), \mathscr{T}^{\prime}\right)$ is a precompact group. Since $\pi(S)$ is dense in $\mathbb{T}^{2^{\tau}}$, we conclude that $\pi(S)$ is $\mathscr{T}^{\prime}$-dense in $\pi(G)$. Since $\pi$ is an isomorphism between $G$ and $\pi(G), \mathscr{T}=\left\{\pi^{-1}(U): U \in \mathscr{T}^{\prime}\right\}$ is the required topology.

The characterization of the countable potentially dense subsets of an abelian group $G$ with $|G| \leq 2^{\mathfrak{c}}$ can be found in $\underline{6}$. Our next corollary shows that Theorem 3.3 is sufficiently useful in obtaining some particular cases of that characterization.

Corollary 3.4. Let $G$ be an unbounded torsion abelian group with $|G| \leq 2^{\mathfrak{c}}$. For a countable subset $S$ of $G$, the following conditions are equivalent:

(i) $S$ is potentially dense in $G$;

(ii) there exists a precompact Hausdorff group topology $\mathscr{T}$ on $G$ such that $S$ is dense in $(G, \mathscr{T})$;

(iii) $S$ is $\omega$-wide.

Proof. The implication (iii) $\rightarrow$ (ii) follows from Theorem 3.3, while the implication (ii) $\rightarrow$ (i) is trivial. According to Proposition 2.2(iii), to prove the implication (i) $\rightarrow$ (iii), it suffices to check that $n S$ is infinite for every $n \in \mathbb{N}$. Assume that $n S$ is finite for some $n \in \mathbb{N}$. By Lemma 3.1(i), this yields that $n G$ is finite as well. Consequently, $G$ is bounded, a contradiction.

In connection with the last corollary, it is worth mentioning that the unboundedness of a torsion abelian group $G$ is a necessary condition for the existence of an $\omega$-wide subset of $G$; see Proposition 2.2(iii). 
Corollary 3.5. Let $G$ be an abelian group such that $|n G|=|G|$ for every $n \in \mathbb{N}$. For an uncountable subset $S$ of $G$, the following conditions are equivalent:

(i) $S$ is potentially dense in $G$;

(ii) $S$ is $\mathscr{T}$-dense in some precompact Hausdorff group topology $\mathscr{T}$ on $G$;

(iii) $\log \log |n G| \leq|n S|$ for every $n \in \mathbb{N}$;

(iv) $\log \log |G| \leq \min \{|n S|: n \in \mathbb{N}\}$.

Proof. The implication (ii) $\rightarrow$ (i) is obvious, the implication (i) $\rightarrow$ (iii) is proved in Corollary 3.2 , and the implication (iii) $\rightarrow$ (iv) holds due to our assumption on $G$. It remains only to check the implication (iv) $\rightarrow$ (ii). From (iv) and Proposition 2.2(i) we conclude that $S$ is $\tau$-wide for $\tau=\log \log |G|$. Since $|G| \leq 2^{2^{\tau}}$, Theorem 3.3 applies.

Corollary 3.6. Let $G$ be an abelian group satisfying one of the following conditions:

(a) $G$ is divisible;

(b) $|t(G)|<|G|$;

(c) $|t(G)| \leq \omega$;

(d) $G$ is almost torsion-free.

For every uncountable subset $S$ of $G$, conditions (i)-(iv) of Corollary 3.5 are equivalent.

Proof. Let $S$ be an uncountable subset of $G$. In particular, $|G| \geq|S|>\omega$. It suffices to show that $G$ satisfies the assumption of Corollary 3.5. Fix $n \in \mathbb{N}$. If (a) holds, then $|n G|=|G|$ trivially holds, as the homomorphism $\eta_{n}: G \rightarrow G$ defined by $\eta_{n}(g)=n g$ for $g \in G$ is surjective. If (b) holds, then from ker $\eta_{n} \subseteq t(G)$ and $|t(G)|<|G|$ it follows that $|n G|=|G|$. If (c) holds, then $t(G) \leq \omega<|G|$, and so (c) is a particular case of (b). Finally, note that every almost torsion-free group satisfies (c).

Remark 3.7. For an uncountable subset $S$ of an abelian group $G$ satisfying items (c) or (d) of Corollary 3.6. the following simplified condition can be added to the list of equivalent items (i)-(iv) of Corollary 3.5.

(v) $\log \log |G| \leq|S|$.

Indeed, since $t(G)$ is at most countable and $S$ is uncountable, we must have $|n S|=|S|$ for every $n \in \mathbb{N}$. This establishes the implication (v) $\rightarrow$ (iv). The reverse implication (iv) $\rightarrow$ (v) is trivial.

Let $S$ be an uncountable subset of an abelian group $G$. Corollaries 3.5 and 3.6 show that (3.1) is not only a necessary but also a sufficient condition for the potential density of $S$ in $G$ when $G$ belongs to a wide class of groups including divisible and (almost) torsion-free groups. According to Remark 3.7, the weaker (and much simpler) condition $\log \log |G| \leq|S|$, obtained by taking $n=1$ in (3.1), is also sufficient for the potential density of $S$ in $G$ when the torsion part $t(G)$ of $G$ is at most countable. Our next example demonstrates that this simpler condition (v) is no longer sufficient for the potential density of $S$ in $G$ when one weakens the assumption $|t(G)| \leq \omega$ to $|t(G)|<|G|$. (Note that $|G| \geq|S|>\omega$.)

Example 3.8. Let $\mathbb{Z}(2)=\mathbb{Z} / 2 \mathbb{Z}$ be the abelian group with two elements. Define $G=\mathbb{Z}(2)^{\omega} \times \mathbb{Z}^{2^{c}}$ and $S=\mathbb{Z}(2)^{\omega} \times C$, where $C$ is any infinite cyclic subgroup of $\mathbb{Z}^{2^{\mathfrak{c}}}$. Then $\log \log |G|=\mathfrak{c}=|S|$ and $|t(G)|=\left|\mathbb{Z}(2)^{\omega} \times\{0\}\right|=\mathfrak{c}<|G|$. Since $\log \log |2 G|>\omega=|2 S|$, the set $S$ cannot be potentially dense in $G$ by Corollary 3.2 . 
Observe that a group $G$ with the properties from Example 3.8 must necessarily have an uncountable torsion part $t(G)$; see Remark 3.7. Example 3.8 provides a negative answer to [4, Question 45].

\section{TECHNiCAL LEMMAS}

We call a connected open subset $V$ of $\mathbb{T}$ an open arc, and we use $l(V)$ to denote the length of $V$.

Lemma 4.1. Suppose that $V$ is an open arc in $\mathbb{T}, z, z^{\prime} \in \mathbb{T}, m, n \in \mathbb{N}, 1 \leq n<m$ and $2 / m<l(V)$. Then there exists $y \in V$ such that $m y=z$ and $n y \neq z^{\prime}$.

Proof. Since $2 / m<l(V)$, the $\operatorname{arc} V$ contains two solutions $y_{0}$ and $y_{1}$ of the equation $m y=z$ with

$$
l\left(C\left(y_{0}, y_{1}\right)\right)=1 / m,
$$

where $C\left(y_{0}, y_{1}\right)$ is the shortest arc in $\mathbb{T}$ connecting $y_{0}$ and $y_{1}$.

Suppose that $n y_{0}=n y_{1}=z^{\prime}$. Then $n\left(y_{0}-y_{1}\right)=0$, and so $l\left(C\left(y_{0}, y_{1}\right)\right) \geq 1 / n$. Together with (4.1), this gives $m \leq n$, a contradiction. Therefore, $n y_{j} \neq z^{\prime}$ for some $j=0,1$, and so we can take that $y_{j}$ as our $y$.

If $G$ and $H$ are groups, then $G \cong H$ means that $G$ and $H$ are isomorphic.

Lemma 4.2. Let $\tau$ and $\kappa$ be infinite cardinals such that $\tau \leq \kappa$. Let $K$ be a subgroup of $\mathbb{T}^{\kappa}$ such that $|K| \leq \tau$. For every $\gamma<\kappa$, let $V_{\gamma}$ be an open arc in $\mathbb{T}$ such that the set $\left\{l\left(V_{\gamma}\right): \gamma<\kappa\right\}$ has a positive lower bound. Then there exists $f \in \prod\left\{V_{\gamma}: \gamma<\kappa\right\}$ having the following properties:

(i) $\langle f\rangle \cong \mathbb{Z}$;

(ii) $\langle f\rangle \cap K=\{0\}$.

Proof. Fix $k \in \mathbb{N}$ such that $2 / k<l\left(V_{\gamma}\right)$ for every $\gamma<\kappa$. Since $|K| \leq \tau$, we can choose an enumeration $K \times \mathbb{N}=\left\{\left(h_{\alpha}, n_{\alpha}\right): \alpha<\tau\right\}$ of the set $K \times \mathbb{N}$. By transfinite recursion on $\alpha<\tau$ we will select $\gamma_{\alpha}<\kappa$ and $y_{\gamma_{\alpha}} \in \mathbb{T}$ satisfying the following properties:

$\left(\mathrm{i}_{\alpha}\right) \gamma_{\alpha} \notin\left\{\gamma_{\beta}: \beta<\alpha\right\}$,

(ii $\left.{ }_{\alpha}\right) y_{\gamma_{\alpha}} \in V_{\gamma_{\alpha}}$,

(iii $\left.{ }_{\alpha}\right) n_{\alpha} y_{\gamma_{\alpha}} \neq h_{\alpha}\left(\gamma_{\alpha}\right)$.

Basis of recursion. Select $\gamma_{0}<\kappa$ arbitrarily. Apply Lemma 4.1 to $V=V_{\gamma_{0}}$, $z=0, z^{\prime}=h_{0}\left(\gamma_{0}\right), n=n_{0}$ and $m=n_{0}(k+1)$ to choose $y_{\gamma_{0}} \in \mathbb{T}$ satisfying (ii ${ }_{0}$ ) and $\left(\mathrm{iii}_{0}\right)$. Condition $\left(\mathrm{i}_{0}\right)$ is vacuous.

Recursive step. Let $\alpha<\tau$, and suppose that $\gamma_{\beta}<\kappa$ and $y_{\gamma_{\beta}} \in \mathbb{T}$ satisfying $\left(\mathrm{i}_{\beta}\right)-\left(\mathrm{iii}_{\beta}\right)$ have already been selected for all $\beta<\alpha$. We will now choose $\gamma_{\alpha}<\kappa$ and $y_{\gamma_{\alpha}} \in \mathbb{T}$ satisfying $\left(\mathrm{i}_{\alpha}\right)-\left(\mathrm{iii}_{\alpha}\right)$. Since $|\alpha|<\tau \leq \kappa$, we can choose $\gamma_{\alpha}<\kappa$ satisfying $\left(\mathrm{i}_{\alpha}\right)$. Now we apply Lemma 4.1 to $V=V_{\gamma_{\alpha}}, z=0, z^{\prime}=h_{\alpha}\left(\gamma_{\alpha}\right), n=n_{\alpha}$ and $m=n_{\alpha}(k+1)$ to choose $y_{\gamma_{\alpha}} \in \mathbb{T}$ satisfying $\left(\mathrm{ii}_{\alpha}\right)$ and ( $\left.\mathrm{iii}_{\alpha}\right)$.

The recursion being complete, choose $y_{\gamma} \in V_{\gamma}$ arbitrarily for every $\gamma \in \kappa \backslash\left\{\gamma_{\alpha}\right.$ : $\alpha<\tau\}$. Define $f \in \mathbb{T}^{\kappa}$ by $f(\gamma)=y_{\gamma}$ for each $\gamma<\kappa$. Then $f \in \prod\left\{V_{\gamma}: \gamma<\kappa\right\}$. We claim that

$$
n f \notin K \quad \text { for every } n \in \mathbb{N} \text {. }
$$


Indeed, let $n \in \mathbb{N}$ and $h \in K$ be arbitrary. Then $(h, n) \in K \times \mathbb{N}$, and so $(h, n)=$ $\left(h_{\alpha}, n_{\alpha}\right)$ for some $\alpha<\kappa$. Now $n f\left(\gamma_{\alpha}\right)=n_{\alpha} y_{\gamma_{\alpha}} \neq h_{\alpha}\left(\gamma_{\alpha}\right)=h\left(\gamma_{\alpha}\right)$ by (iii $\left.{ }_{\alpha}\right)$. Thus, $n f \neq h$. Since $h \in K$ was arbitrary, this proves (4.2). From (4.2) we immediately get both (i) and (ii).

Lemma 4.3. Let $\tau$ and $\kappa$ be infinite cardinals such that $\tau \leq \kappa$. Let $K$ be a subgroup of $\mathbb{T}^{\kappa}$ such that $|K| \leq \tau$. Assume that $f^{\prime} \in K, m \in \mathbb{N}$ and $m \geq 2$. For every $\gamma<\kappa$, let $V_{\gamma}$ be an open arc in $\mathbb{T}$ such that $2 / m<l\left(V_{\gamma}\right)$. Then there exists $f \in \prod\left\{V_{\gamma}: \gamma<\kappa\right\}$ satisfying the following properties:

(i) $m f=f^{\prime}$;

(ii) $n f \notin K$ for all $n \in \mathbb{N}$ with $1 \leq n<m$.

Proof. Since $|K| \leq \tau$, we can choose an enumeration $K \times\{1,2, \ldots, m-1\}=$ $\left\{\left(h_{\alpha}, n_{\alpha}\right): \alpha<\tau\right\}$ of the set $K \times\{1,2, \ldots, m-1\}$. By transfinite recursion on $\alpha<\tau$ we will select $\gamma_{\alpha}<\kappa$ and $y_{\gamma_{\alpha}} \in \mathbb{T}$ with the following properties:

(i $\left.\mathrm{i}_{\alpha}\right) \gamma_{\alpha} \notin\left\{\gamma_{\beta}: \beta<\alpha\right\}$,

(ii $\left.{ }_{\alpha}\right) y_{\gamma_{\alpha}} \in V_{\gamma_{\alpha}}$,

(iii $\left.\alpha_{\alpha}\right) m y_{\gamma_{\alpha}}=f^{\prime}\left(\gamma_{\alpha}\right)$,

$\left(\operatorname{iv}_{\alpha}\right) n_{\alpha} y_{\gamma_{\alpha}} \neq h_{\alpha}\left(\gamma_{\alpha}\right)$.

Basis of recursion. Select $\gamma_{0}<\kappa$ arbitrarily, and apply Lemma 4.1 to $V=V_{\gamma_{0}}$, $z=f^{\prime}\left(\gamma_{0}\right), z^{\prime}=h_{0}\left(\gamma_{0}\right), n=n_{0}$ and $m$ to choose $y_{\gamma_{0}} \in \mathbb{T}$ satisfying $\left(\mathrm{ii}_{0}\right)$, (iii $)$ and $\left(\mathrm{iv}_{0}\right)$. Condition $\left(\mathrm{i}_{0}\right)$ is vacuous.

Recursive step. Let $\alpha<\tau$, and suppose that $\gamma_{\beta}<\kappa$ and $y_{\gamma_{\beta}} \in \mathbb{T}$ satisfying $\left(\mathrm{i}_{\beta}\right)-\left(\mathrm{iv}_{\beta}\right)$ have already been selected for all $\beta<\alpha$. We will now choose $\gamma_{\alpha}<\kappa$ and $y_{\gamma_{\alpha}} \in \mathbb{T}$ satisfying $\left(\mathrm{i}_{\alpha}\right)-\left(\mathrm{iv}_{\alpha}\right)$. Since $|\alpha|<\tau \leq \kappa$, we can choose $\gamma_{\alpha}<\kappa$ satisfying $\left(\mathrm{i}_{\alpha}\right)$. Now we apply Lemma 4.1 to $V=V_{\gamma_{\alpha}}, z=f^{\prime}\left(\gamma_{\alpha}\right), z^{\prime}=h_{\alpha}\left(\gamma_{\alpha}\right), n=n_{\alpha}$ and $m$ to choose $y_{\gamma_{\alpha}} \in \mathbb{T}$ satisfying $\left(\mathrm{ii}_{\alpha}\right),\left(\mathrm{iii}_{\alpha}\right)$ and $\left(\mathrm{iv}_{\alpha}\right)$.

The recursion being complete, for every $\gamma \in \kappa \backslash\left\{\gamma_{\alpha}: \alpha<\tau\right\}$, apply Lemma 4.1 to $V=V_{\gamma}, z=f^{\prime}(\gamma), z^{\prime}=0, n=1$ and $m$ to choose $y_{\gamma} \in V_{\gamma}$ such that $m y_{\gamma}=f^{\prime}(\gamma)$.

Define $f \in \mathbb{T}^{\kappa}$ by $f(\gamma)=y_{\gamma}$ for every $\gamma<\kappa$. Then $f \in \prod\left\{V_{\gamma}: \gamma<\kappa\right\}$ and (i) is satisfied. To prove (ii), choose $n \in \mathbb{N}$ such that $1 \leq n<m$. Let $h \in K$ be arbitrary. Then $(h, n) \in K \times\{1,2, \ldots, m-1\}$, and so $(h, n)=\left(h_{\alpha}, n_{\alpha}\right)$ for some $\alpha<\kappa$. Now $n f\left(\gamma_{\alpha}\right)=n_{\alpha} y_{\gamma_{\alpha}} \neq h_{\alpha}\left(\gamma_{\alpha}\right)=h\left(\gamma_{\alpha}\right)$ by (iv $\left.{ }_{\alpha}\right)$. Therefore, $n f \neq h$. Since $h \in K$ was arbitrary, this proves $n f \notin K$.

Lemma 4.4. Let $G$ and $G^{*}$ be abelian groups, and let $K$ and $K^{*}$ be subgroups of $G$ and $G^{*}$, respectively. Suppose also that $x \in G, x^{*} \in G^{*}, m \in \mathbb{N}, m \geq 2$, and $\psi: K \rightarrow K^{*}$ is an isomorphism satisfying the following properties:

(a) $m x \in K$ and $m x^{*} \in K^{*}$;

(b) $n x \notin K$ and $n x^{*} \notin K^{*}$ whenever $n \in \mathbb{N}$ and $1 \leq n<m$;

(c) $\psi(m x)=m x^{*}$.

Then there exists a unique isomorphism $\varphi: K+\langle x\rangle \rightarrow K^{*}+\left\langle x^{*}\right\rangle$ extending $\psi$ such that $\varphi(x)=x^{*}$.

Proof. Define $\varphi$ by

$$
\varphi(h+k x)=\psi(h)+k x^{*} \text { for } h \in K \text { and } k \in \mathbb{Z} .
$$

To check that this definition is correct, suppose that

$$
h+k x=h^{\prime}+k^{\prime} x
$$


with $h, h^{\prime} \in K$ and $k, k^{\prime} \in \mathbb{Z}$. Hence, $\left(k^{\prime}-k\right) x=h-h^{\prime} \in K$, so by (a) and (b), one has $k^{\prime}-k=l m$ for some $l \in \mathbb{Z}$, which yields $h-h^{\prime}=l m x$. This gives

$\psi(h)-\psi\left(h^{\prime}\right)=\psi\left(h-h^{\prime}\right)=\psi(\operatorname{lm} x)=l \psi(m x)=l m x^{*}=\left(k^{\prime}-k\right) x^{*}=k^{\prime} x^{*}-k x^{*}$

by (c), and so $\psi(h)+k x^{*}=\psi\left(h^{\prime}\right)+k^{\prime} x^{*}$. Comparing this with (4.4), we conclude that (4.3) correctly defines a homomorphism $\varphi$. From (4.3) we get $\varphi(x)=x^{*}$ and $\varphi \uparrow_{K}=\psi$. Moreover, $\varphi$ is surjective and unique with these properties.

To prove that $\varphi$ is a monomorphism, assume that $\varphi(h+k x)=0$ for some $h \in K$ and $k \in \mathbb{Z}$. Then $\psi(h)+k x^{*}=0$, so $k x^{*} \in K^{*}$. Consequently, $m$ divides $k$ by (a) and (b). Then $k x \in K$, and so $h+k x \in K$ as well. Therefore, $\varphi(h+k x)=\psi(h+k x)=0$ yields $h+k x=0$, as $\psi$ is a monomorphism.

\section{Proof of Theorem 2.3}

Fix a countable base $\mathscr{V}$ for the topology of $\mathbb{T}$ consisting of non-empty open arcs of $\mathbb{T}$ such that $\mathbb{T} \in \mathscr{V}$. Consider the Tychonoff product topology on $2^{\tau}$, and let $\mathscr{B}$ be the canonical base for $2^{\tau}$ consisting of non-empty clopen subsets of $2^{\tau}$ such that $|\mathscr{B}|=\tau$. Let

$$
\mathbb{U}=\left\{\mathscr{U} \in[\mathscr{B}]^{<\omega}: \mathscr{U} \text { is a cover of } 2^{\tau} \text { by pairwise disjoint sets }\right\} \text {. }
$$

For $\xi \in 2^{\tau}$ and $\mathscr{U} \in \mathbb{U}$, let $U_{\xi, \mathscr{U}} \in \mathscr{U}$ denote the unique $U \in \mathscr{U}$ such that $\xi \in U$. Define

$$
\mathbb{E}=\{(\mathscr{U}, v): \mathscr{U} \in \mathbb{U} \text { and } v: \mathscr{U} \rightarrow \mathscr{V} \text { is a function }\} .
$$

For $(\mathscr{U}, v) \in \mathbb{E}$, let

$$
F(\mathscr{U}, v)=\left\{f \in \mathbb{T}^{2^{\tau}}: f(\xi) \in v\left(U_{\xi, \mathscr{U}}\right) \text { for all } \xi \in 2^{\tau}\right\}=\prod\left\{v\left(U_{\xi, \mathscr{U}}\right): \xi \in 2^{\tau}\right\} .
$$

Clearly, $|\mathbb{E}|=\tau$, so we can fix an enumeration $\mathbb{E}=\left\{\left(\mathscr{U}_{\alpha}, v_{\alpha}\right): \alpha<\tau\right\}$ of $\mathbb{E}$ such that $\mathscr{U}_{0}=\left\{2^{\tau}\right\}$ and $v_{0}\left(2^{\tau}\right)=\mathbb{T}$. For each $\alpha<\tau$, choose $n_{\alpha} \in \mathbb{N}$ such that

$$
2 / n_{\alpha}<\min \{l(v(U)): U \in \mathscr{U}\} .
$$

By transfinite recursion on $\alpha<\tau$ we will choose an element $x_{\alpha} \in S$ and define a map $\varphi_{\alpha}: H_{\alpha}=\left\langle\left\{x_{\beta}: \beta \leq \alpha\right\}\right\rangle \rightarrow \mathbb{T}^{2^{\tau}}$ satisfying the following conditions:

$\left(\mathrm{i}_{\alpha}\right) \varphi_{\alpha}\left(x_{\alpha}\right) \in F\left(\mathscr{U}_{\alpha}, v_{\alpha}\right)$,

(ii $\left.\alpha_{\alpha}\right) \varphi_{\alpha}$ is a monomorphism,

(iii $\alpha) \varphi_{\alpha}\left\lceil_{H_{\beta}}=\varphi_{\beta}\right.$ for all $\beta<\alpha$.

Basis of recursion. Pick $x_{0} \in S$ arbitrarily, and let $\varphi_{0}:\left\langle x_{0}\right\rangle \rightarrow \mathbb{T}^{2^{\tau}}=F\left(\mathscr{U}_{0}, v_{0}\right)$ be an arbitrary monomorphism. Then conditions $\left(\mathrm{i}_{0}\right)$ and $\left(\mathrm{ii}_{0}\right)$ are satisfied, while the condition ( $\left.\mathrm{iii}_{0}\right)$ is vacuous.

Recursive step. Let $\alpha<\tau$, and suppose that $x_{\beta} \in S$ and a map $\varphi_{\beta}: H_{\beta} \rightarrow \mathbb{T}^{2^{\tau}}$ satisfying $\left(\mathrm{i}_{\beta}\right),\left(\mathrm{ii}_{\beta}\right)$ and $\left(\mathrm{iii}_{\beta}\right)$ have already been constructed for every $\beta<\alpha$. We are going to define $x_{\alpha} \in S$ and a map $\varphi_{\alpha}: H_{\alpha} \rightarrow \mathbb{T}^{2^{\tau}}$ satisfying $\left(\mathrm{i}_{\alpha}\right)$, (ii $\left.{ }_{\alpha}\right)$ and $\left(\right.$ iii $\left._{\alpha}\right)$.

Define

$$
H_{\alpha}^{\prime}=\left\langle\left\{x_{\beta}: \beta<\alpha\right\}\right\rangle=\bigcup_{\beta<\alpha} H_{\beta} .
$$

Since $\left(\mathrm{ii}_{\beta}\right)$ and $\left(\mathrm{iii}_{\beta}\right)$ hold for every $\beta<\alpha$,

$$
\varphi_{\alpha}^{\prime}=\bigcup_{\beta<\alpha} \varphi_{\beta}: H_{\alpha}^{\prime} \rightarrow \mathbb{T}^{2^{\tau}}
$$


is a monomorphism. Since $\left\{x_{\beta}: \beta<\alpha\right\} \subseteq S,|\alpha|<\tau$ and $S$ is $\tau$-wide, one has $n_{\alpha} ! S \backslash\left\langle\left\{x_{\beta}: \beta<\alpha\right\}\right\rangle=n_{\alpha} ! S \backslash H_{\alpha}^{\prime} \neq \emptyset$, and so there exists $x_{\alpha} \in S$ such that $n_{\alpha} ! x_{\alpha} \notin H_{\alpha}^{\prime}$. In particular,

$$
n x_{\alpha} \notin H_{\alpha}^{\prime} \text { for all } n \leq n_{\alpha} .
$$

Let $\kappa=2^{\tau}$ and $K=\varphi_{\alpha}^{\prime}\left(H_{\alpha}^{\prime}\right)$. For $\xi \in 2^{\tau}$, define $V_{\xi}=v_{\alpha}\left(U_{\xi, \mathscr{U}_{\alpha}}\right)$. Then (5.1) yields

$$
2 / n_{\alpha}<l\left(v_{\alpha}\left(U_{\xi, \mathscr{U}_{\alpha}}\right)\right)=l\left(V_{\xi}\right) \quad \text { for every } \quad \xi \in 2^{\tau} .
$$

We need to consider two cases.

Case 1. $\left\{n \in \mathbb{N}: n x_{\alpha} \in H_{\alpha}^{\prime}\right\}=\emptyset$. In this case, $\left\langle x_{\alpha}\right\rangle \cong \mathbb{Z}$ and the sum $\left\langle x_{\alpha}\right\rangle+H_{\alpha}^{\prime}=$ $\left\langle x_{\alpha}\right\rangle \oplus H_{\alpha}^{\prime}$ is direct. Since $\left\{l\left(V_{\gamma}\right): \gamma<\kappa\right\}$ has a positive lower bound by (5.3), we can apply Lemma 4.2 to choose

$$
f \in \prod\left\{V_{\xi}: \xi \in 2^{\tau}\right\}=F\left(\mathscr{U}_{\alpha}, v_{\alpha}\right)
$$

with $\langle f\rangle \cong \mathbb{Z}$ and $\langle f\rangle \cap K=\{0\}$. Then the sum $K+\langle f\rangle=K \oplus\langle f\rangle$ is direct as well. Since $\left\langle x_{\alpha}\right\rangle \cong\langle f\rangle \cong \mathbb{Z}$, there exists a unique monomorphism $\varphi_{\alpha}: H_{\alpha}=$ $H_{\alpha}^{\prime} \oplus\left\langle x_{\alpha}\right\rangle \rightarrow K \oplus\langle f\rangle \subseteq \mathbb{T}^{2^{\tau}}$ extending $\varphi_{\alpha}^{\prime}$ such that $\varphi_{\alpha}\left(x_{\alpha}\right)=f$.

Case 2. $\left\{n \in \mathbb{N}: n x_{\alpha} \in H_{\alpha}^{\prime}\right\} \neq \emptyset$. Let $m=\min \left\{n \in \mathbb{N}: n x_{\alpha} \in H_{\alpha}^{\prime}\right\}$ and $f^{\prime}=$ $\varphi_{\alpha}^{\prime}\left(m x_{\alpha}\right) \in K$. Then $m>n_{\alpha}$ by (5.2), so from (5.3) we obtain $2 / m<2 / n_{\alpha}<l\left(V_{\xi}\right)$ for every $\xi \in 2^{\tau}$. Since $n_{\alpha} \geq 1$, we have $m \geq 2$. Applying Lemma 4.3. we get $f$ satisfying (5.4) such that $m f=f^{\prime}$ and $n f \notin K$ for all $n \in \mathbb{N}$ with $1 \leq n<m$. Observe that $\mathbb{T}^{2^{\tau}}$ (taken as $\left.G^{*}\right), H_{\alpha}^{\prime}($ taken as $K), K$ (taken as $\left.K^{*}\right), x_{\alpha}$ (taken as $x$ ), $f$ (taken as $\left.x^{*}\right), \varphi_{\alpha}^{\prime}$ (taken as $\psi$ ) and $m$ satisfy the assumptions of Lemma 4.4 . Denoting $\varphi$ from the conclusion of this lemma by $\varphi_{\alpha}$, we obtain a monomorphism $\varphi_{\alpha}: H_{\alpha} \rightarrow \mathbb{T}^{2^{\tau}}$ extending $\varphi_{\alpha}^{\prime}$ such that $\varphi_{\alpha}\left(x_{\alpha}\right)=f$.

The monomorphism $\varphi_{\alpha}$ obviously satisfies $\left(\mathrm{i}_{\alpha}\right),\left(\mathrm{ii}_{\alpha}\right)$ and $\left(\mathrm{iii}_{\alpha}\right)$ in both cases.

The recursive construction being complete, let

$$
H=\bigcup_{\alpha<\tau} H_{\alpha} \quad \text { and } \quad \varphi=\bigcup_{\alpha<\tau} \varphi_{\alpha} .
$$

Since $\left(\mathrm{ii}_{\alpha}\right)$ and $\left(\mathrm{iii}_{\alpha}\right)$ are satisfied for every $\alpha<\tau, \varphi: H \rightarrow \mathbb{T}^{2^{\tau}}$ is a monomorphism.

It remains only to check that $\varphi(H \cap S)$ is dense in $\mathbb{T}^{2^{\tau}}$. Let $W$ be a non-empty open subset of $\mathbb{T}^{2^{\tau}}$. Choose pairwise distinct $\xi_{1}, \ldots, \xi_{i} \in 2^{\tau}$ and $V_{1}, \ldots, V_{i} \in \mathscr{V}$ such that

$$
\left\{f \in \mathbb{T}^{2^{\tau}}: f\left(\xi_{j}\right) \in V_{j} \text { for all } j \leq i\right\} \subseteq W .
$$

Select $\mathscr{U}=\left\{U_{1}, \ldots, U_{i}\right\} \in \mathbb{U}$ which separates the $\xi_{j}$ 's; that is, $j, k \leq i$ and $\xi_{j} \in U_{k}$ imply $j=k$. Define $v: \mathscr{U} \rightarrow \mathscr{V}$ by $v\left(U_{j}\right)=V_{j}$ for $j \leq i$. Then $(\mathscr{U}, v) \in \mathbb{E}$, and so $(\mathscr{U}, v)=\left(\mathscr{U}_{\alpha}, v_{\alpha}\right)$ for some $\alpha<\tau$. Note that

$$
F\left(\mathscr{U}_{\alpha}, v_{\alpha}\right)=F(\mathscr{U}, v) \subseteq\left\{f \in \mathbb{T}^{2^{\tau}}: f\left(\xi_{j}\right) \in V_{j} \text { for all } j \leq i\right\} \subseteq W .
$$

Since $x_{\alpha} \in S \cap H_{\alpha} \subseteq H \cap S$ and $\varphi\left(x_{\alpha}\right)=\varphi_{\alpha}\left(x_{\alpha}\right) \in F\left(\mathscr{U}_{\alpha}, v_{\alpha}\right)$ by $\left(\mathrm{i}_{\alpha}\right)$, it follows from (5.5) that $\varphi\left(x_{\alpha}\right) \in \varphi(H \cap S) \cap W \neq \emptyset$. 


\section{Questions}

All instances of potential density in Section 3 were witnessed by a precompact Hausdorff group topology. This makes it natural to ask the following question.

Question 6.1. Let $S$ be a potentially dense subset of an abelian group $G$. Does there exist a Hausdorff precompact group topology $\mathscr{T}$ on $G$ such that $S$ is $\mathscr{T}$-dense in $G$ ?

The answer to this question is positive when $S$ is countable [6].

It was shown in [5] that every potentially dense subset $S$ of an abelian group $G$ must be Zariski dense in $G$. (We refer the reader to the Introduction for the definition of Zariski density.) Corollary 3.2 gives another necessary condition for the potential density of $S$ in $G$. One may wonder if these two conditions, combined together, are also sufficient.

Question 6.2. Let $S$ be an infinite subset of an Abelian group $G$ such that:

(a) $S$ is Zariski dense in $G$, and

(b) $\log \log |n G| \leq|n S|$ (equivalently, $|n G| \leq 2^{2^{|n S|}}$ ) for all $n \in \mathbb{N}$.

Is $S$ potentially dense in $G$ ? Does there exist a Hausdorff precompact group topology $\mathscr{T}$ on $G$ such that $S$ is $\mathscr{T}$-dense in $G$ ?

We note that this question is an appropriate modification of [4, Question 45], which is necessary in view of Example 3.8 .

\section{ACKNOWLEDGMENT}

It is our pleasure to thank the referee for useful suggestions that inspired the authors to redesign Section 3.

\section{REFERENCES}

1. D. N. Dikranjan, I. R. Prodanov, and L. N. Stoyanov, Topological Groups (Characters, Dualities and Minimal Group Topologies), Monographs and Textbooks in Pure and Applied Mathematics, 130, Marcel Dekker, Inc., New York-Basel, 1990. MR1015288 (91e:22001)

2. D. Dikranjan and D. Shakhmatov, Algebraic structure of pseudocompact groups, Mem. Amer. Math. Soc. 133 (1998), 83 pages. MR1396956 (98j:22001)

3. D. Dikranjan and D. Shakhmatov, Forcing hereditarily separable compact-like group topologies on abelian groups, Topology Appl. 151 (2005), 2-54. MR2139740(2006d:22002)

4. D. Dikranjan and D. Shakhmatov, Selected topics from the structure theory of topological groups, pp. 389-406 in: Open Problems in Topology. II (E. Perl, ed.), Elsevier, 2007.

5. D. Dikranjan and D. Shakhmatov, The Markov-Zariski topology of an abelian group, J. Algebra, to appear.

6. D. Dikranjan and D. Shakhmatov, A Kronecker-Weyl theorem for subsets of Abelian groups, submitted.

7. D. Dikranjan and M. Tkačenko, Weakly complete free topological groups, Topology Appl. 112 (2001), 259-287. MR:1824163 (2002b:22004)

8. R. Engel'king, General topology (second edition), Sigma Series in Pure Mathematics, 6, Heldermann Verlag, Berlin, 1989. MR1039321 (91c:54001)

9. A. A. Markov, On unconditionally closed sets (Russian), Mat. Sbornik 18 (1946), 3-28; English translation in: A. A. Markov, Three papers on topological groups: I. On the existence of periodic connected topological groups, II. On free topological groups, III. On unconditionally closed sets, Amer. Math. Soc. Translation 1950 (1950), no. 30, 120 pp.; another English translation in: Topology and Topological Algebra, Translations Series 1, vol. 8, pp. 273-304, Amer. Math. Soc., 1962. MR0015395 (7:412b) MR0037854 (12:318b) 
10. M. Tkachenko and I. Yaschenko, Independent group topologies on abelian groups, Topology Appl. 122 (2002), 425-451. MR1919318 (2003h:22003)

11. H. Weyl, Über die Gleichverteilung von Zahlen mod. Eins (German), Math. Ann. 77 (1916), no. 3, 313-352. MR.1511862

Università di Udine, Dipartimento di Matematica e Informatica, via delle Scienze, 206 - 33100 Udine, ITALY

E-mail address: dikran.dikranjan@dimi.uniud.it

Division of Mathematics, Physics and Earth Sciences, Graduate School of Science And Engineering, Ehime University, Matsuyama 790-8577, Japan

E-mail address: dmitri@dpc.ehime-u.ac.jp 